\title{
Increased Wall Enhancement Extent Representing Higher Rupture Risk of Unruptured Intracranial Aneurysms
}

\author{
Yeqing Jiang, ${ }^{1, *}$ Feng Xu, ${ }^{2, *}$ Lei Huang, ${ }^{1}$ Gang Lu, Liang Ge, Hailin Wan, ${ }^{1}$ Daoying Geng, ${ }^{1}$ Xiaolong Zhang ${ }^{1}$ \\ Department of Radiology, Huashan Hospital Affiliated to Fudan University, Shanghai, China \\ Department of Neurosurgery, ${ }^{2}$ Huashan Hospital Affiliated to Fudan University, Shanghai, China
}

Objective : This study aims to investigate the relationship between aneurysm wall enhancement and clinical rupture risks based on the magnetic resonance vessel wall imaging (MR-VWI) quantitative methods.

Methods : One hundred and eight patients with 127 unruptured aneurysms were prospectively enrolled from Feburary 2016 to October 2017. Aneurysms were divided into high risk $(\geq 10)$ and intermediate-low risk group $(<10)$ according to the PHASES (Population, Hypertension, Age, Size of aneurysm, Earlier SAH history from another aneurysm, Site of aneurysm) scores. Clinical risk factors, aneurysm morphology, and wall enhancement index (WEI) calculated using 3D MR-VWI were analyzed and compared.

Results : In comparison of high-risk and intermediated-low risk groups, univariate analysis showed that neck width $(4.5 \pm 3.3 \mathrm{~mm}$ vs. $3.4 \pm 1.7 \mathrm{~mm}, p=0.002)$, the presence of wall enhancement ( $100.0 \%$ vs. $62.9 \%, p<0.001)$, and WEI $(1.6 \pm 0.6 \mathrm{vs.} 0.8 \pm 0.8, p<0.001)$ were significantly associated with high rupture risk. Multivariate regression analysis revealed that WEl was the most important factor in predicting high rupture risk (odds ratio, 2.6; $95 \%$ confidence interval, 1.4-4.9; $p=0.002$ ). The receiver operating characteristic (ROC) curve analysis can efficiently differentiate higher risk aneurysms (area under the curve, $0.780 ; p<0.001$ ) which have a reliable WEl cutoff value (1.04; sensitivity, 0.833 ; specificity, 0.67$)$ predictive of high rupture risk.

Conclusion : Aneurysms with higher rupture risk based on PHASES score demonstrate increased neck width, wall enhancement, and the enhancement intensity. Higher WEl in unruptured aneurysms has a predictive value for increased rupture risk.

Key Words : Intracranial aneurysm, Unruptured · Vessel wall MRI · Natural history · Rupture risk.

\section{INTRODUCTION}

Unruptured intracranial aneurysms are relatively common vascular lesions, represents $3-5 \%$ of the adult population worldwide ${ }^{28)}$. Unruptured aneurysms often remain asymptomatic. Once rupture occurs, they usually result in devastat-

- Received : May 19, 2020 •Revised : June 25, 2020 •Accepted : July 20, 2020

- Address for reprints : Daoying Geng

Department of Radiology, Huashan Hospital Affiliated to Fudan University, Wulumuqi Middle Road No.12, Jing'an District, Shanghai 200040, China

Tel : +86-13918539866, Fax : +86-13918539866, E-mail : gengdy@163.com, ORCID : https://orcid.org/0000-0002-1707-1521

\section{Xiaolong Zhang}

Department of Radiology, Huashan Hospital Affiliated to Fudan University, Wulumuqi Middle Road No.12, Jing'an District, Shanghai 200040, China

Tel : +86-52888761, Fax : +86-52888764, E-mail : xiaolongzhang@fudan.edu.cn, ORCID : https://orcid.org/0000-0002-1206-5413

*These authors contributed equally to this work.

This is an Open Access article distributed under the terms of the Creative Commons Attribution Non-Commercial License (http://creativecommons.org/licenses/by-nc/4.0) which permits unrestricted non-commercial use, distribution, and reproduction in any medium, provided the original work is properly cited. 
ing subarachnoid hemorrhage with a high mortality and morbidity. On the other hand, potentially serious complications related to treatments should not be neglected. Thus, identifying reliable indicators of risk for aneurysm rupture is crucial for clinical decision making. The PHASES (Population, Hypertension, Age, Size of aneurysm, Earlier SAH history from another aneurysm, Site of aneurysm) score, a clinical prediction model based on patient characteristics and aneurysm morphology, was proposed for assessing the rupture risk ${ }^{3,9)}$. However, this score does not take into account physiological changes of the aneurysmal wall.

Recently, magnetic resonance vessel wall imaging (MRVWI) has emerged as a valuable noninvasive tool for assessing aneurysmal wall pathology. Aneurysm wall enhancement (AWE) has been suggested as a specific marker of unstable or ruptured aneurysms ${ }^{7,11,24)}$. However, the association between the AWE and risk predictors of aneurysms rupture, like the PHASES score, is not well described. Therefore, this study aims to investigate the AWE extent detected on quantitative MR-VWI and clinical risk factors to predict high rupture risk of unruptured aneurysms based on the PHASES scores.

\section{MATERIALS AND METHODS}

\section{Population}

This study was approved by the Institutional Review Board of Huashan Hospital Affiliated to Fudan University (IRB No. KY2019-009). Patients with unruptured intracranial aneurysms diagnosed by 3D MR-VWI were prospectively enrolled in our center from February 2016 to October 2017. The exclusion criteria were the following : 1) lack of MR-VWI examina-
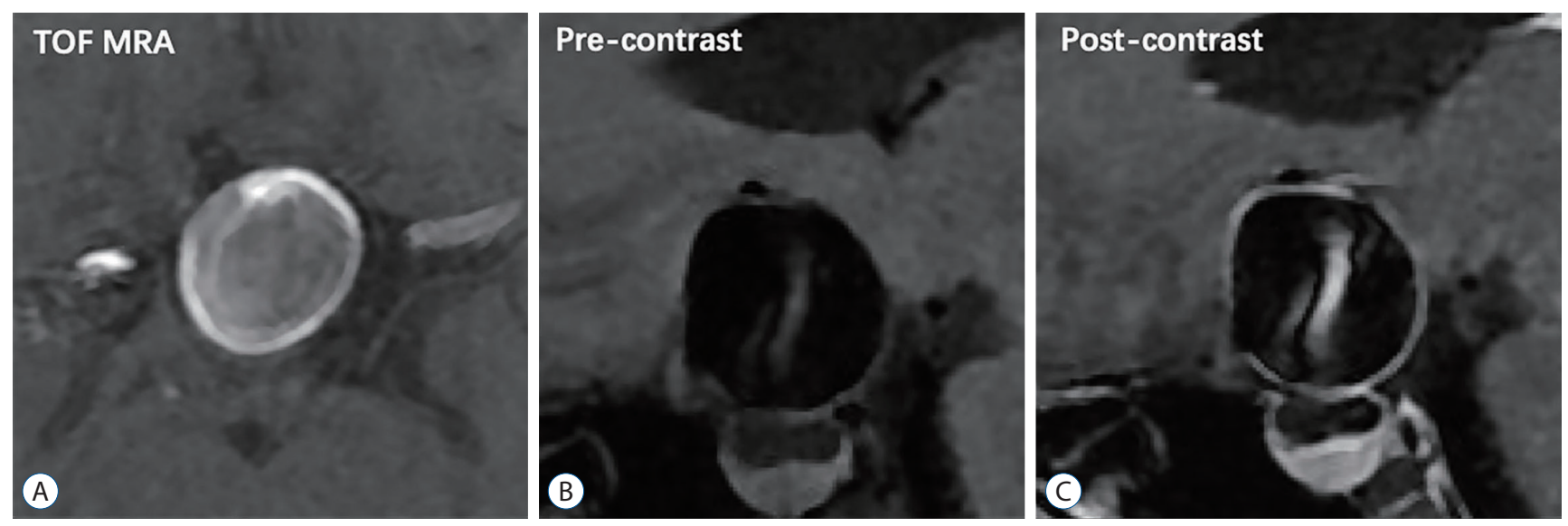

Fig. 1. Three different MR imaging sequences : with $\times 400 \%$ zoomed images. A : TOF MRA. B : Sag CUBE T1 pre-gadolinium. C : Sag CUBE T1 postgadolinium. MRA : magnetic resonance angiography, TOF : time-of-flight.
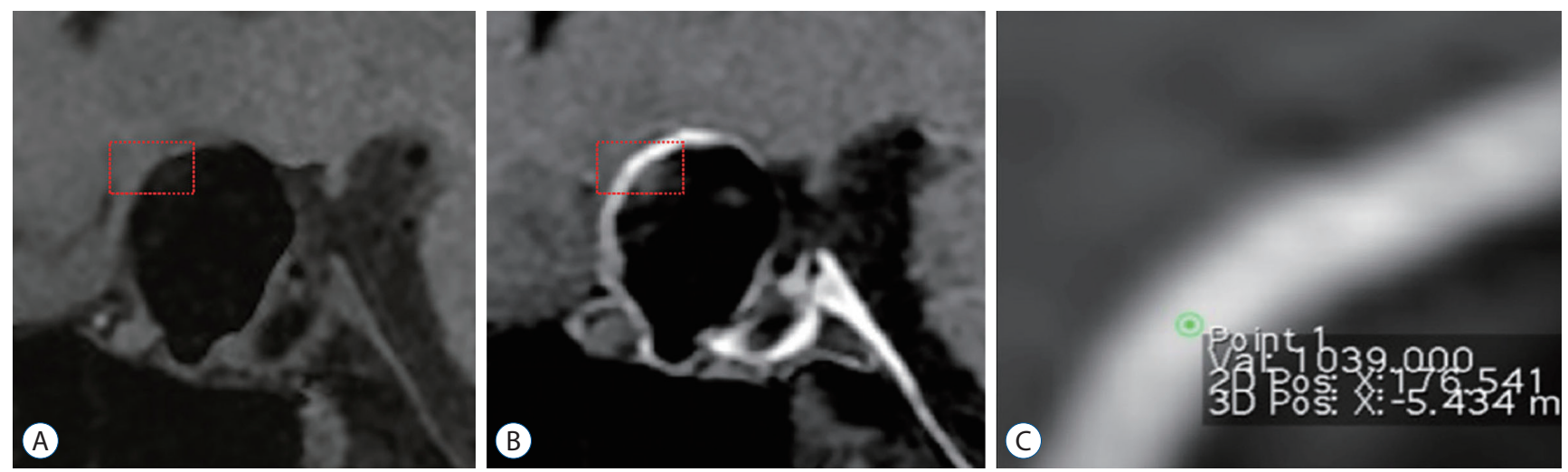

Fig. 2. Representing case. Measurement of signal value (red box). A : Pre-contrast imaging. B : Post-contrast imaging. C : Selected a region of maximum signal using ring tool. 
tion, 2) dissecting or fusiform aneurysms confirmed by MRVWI, 3) a previously treated aneurysm, and 4) insufficient magnetic resonance imaging (MRI) imaging quality to observe aneurysm wall characteristics. Patients' age, gender, risk factors (such as hypertension, earlier subarachnoid hemorrhage (SAH) from another aneurysm), examination time and clinical manifestations were obtained from medical history.

\section{Imaging protocol}

All patients were consecutively scanned utilizing a 3T MR scanner (Discovery MR750; GE Healthcare, Milwaukee, WI, USA) with 32-channel head coil. The images from three different MR scanning sequences were investigated as shown in Fig. 1 with 400\% enlargement. As shown in the enlarged image of the aneurysm in Fig. 1C, the sac wall from the 3D CUBE T1 was significantly highlighted by the contrast agent.
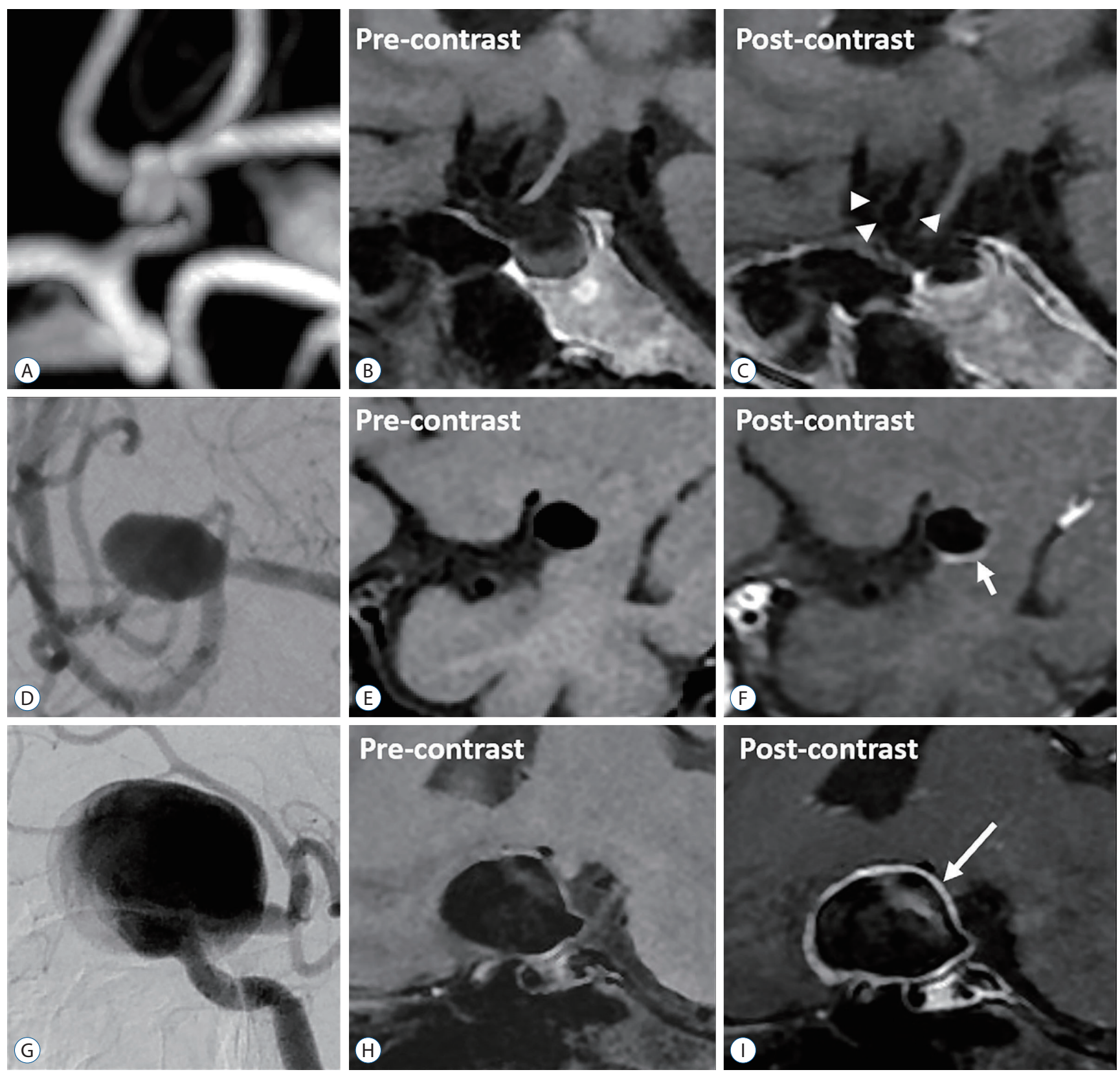

Fig. 3. Representative pre- and post-contrast imaging of aneurysm with different wall enhancement types. A-C : No enhancement (arrowheads). D-F : Partial enhancement (short arrow). G-I : circumferential enhancement (long arrow). 
Location and maximum diameter were obtained through 3Dtime-of-flight (TOF)-MR angiography (MRA) reconstruction images. The imaging protocols included 3D TOF MRA sequence, T1-weighted 3D CUBE fast-spin-echo sequence (field of view, $20 \times 20 \times 16 \mathrm{~cm}^{3}$; repetition time/echo time, $600 / 13.7$ ms; matrix, $288 \times 288 \times 160$ interpolated to $512 \times 512 \times 320$; spatial resolution, $0.4 \times 0.4 \times 0.4 \mathrm{~mm}$; section thickness, $1.0 \mathrm{~mm}$; pixel bandwidth, $62.5 \mathrm{kHz}$; echo chain length, 24; acquisition time, 256 seconds) before and after administration of Gd-BTDO3A (Gadovist, Bayer Schering Pharma, Berlin, Germany) at $0.1 \mathrm{mmol} / \mathrm{kg}$. All raw data were transferred to GE workstation for analysis using the same location at all 3D sequences to ensure data comparability.

\section{PHASES score}

The risk of aneurysm rupture in Chinese people is assumed to be similar to Japanese. Thus, populations from China had three points for geographical region ${ }^{5,14)}$. PHASES risk scores in this dataset were divided into two levels based on the rupture risk (high risk $\geq 10$ points, intermediate-low risk $<10$ points).

\section{Morphology}

Aneurysm morphological characteristics recorded included : 1) neck width (the largest cross sectional diameter of the aneurysm neck); 2) presence of daughter sac (defined as being
$<50 \%$ of the parent aneurysm size); and 3) presence of multiple lobes (defined as being $>50 \%$ of the parent aneurysm

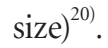

\section{Wall enhancement extent and type}

The WEI was measured quantitatively through 3D CUBE T1 sequencing pre- and post-contrast enhancement ${ }^{22)}$. Aneurysm wall signal acquisition was obtained using the workstation software ring signal tool, selecting the most obvious AWE and the maximum signal (Fig. 2). Reference signal was acquired from a specific location in the right frontal lobe white matter with T1 weighted image (T1WI) sequence pre- and post-enhancement. Signal intensity of the aneurysm wall was calculated as wall signal divided by the same sequence reference signal.

$\mathrm{WEI}=$ (enhanced T1WI wall signal intensity - unenhanced T1WI wall signal intensity) / unenhanced T1WI wall signal intensity

Wall enhancement types were classified into three groups : circumferential, partial, and no enhancement (Fig. 3).

\section{Statistical analysis}

SPSS ver. 22 software (SPSS Inc., Chicago, IL, USA) was used for statistical analysis, and $p<0.05$ was considered statistically significant. The continuous variables were described as

Table 1. PHASES predictors distribution between high and intermediate-low risk group

\begin{tabular}{lcc}
\hline PHASES predictor & High-risk $(\mathbf{n = 3 0 )}$ & Intermediate-low $(\mathbf{n}=\mathbf{9 7})$ \\
\hline Hypertension & $21(70.0)$ & $53(54.6)$ \\
Age (years) & $59.2 \pm 9.0$ & $58.2 \pm 14.2$ \\
$\geq 70$ & $5(17.9)$ & $10(10.4)$ \\
Earlier SAH history & $8(26.7)$ & $12(12.4)$ \\
Size $($ mm) & $12.7 \pm 4.9$ & $5.3 \pm 2.7$ \\
$<7.0$ & $3(10.0)$ & $79(81.4)$ \\
$7.0-9.9$ & $4(13.3)$ & $15(15.5)$ \\
$10.0-19.9$ & $20(66.7)$ & $3(3.1)$ \\
$\geq 20$ & $3(10.0)$ & $0(0.0)$ \\
Location & & $34(35.1)$ \\
ICA & $8(26.7)$ & $25(25.8)$ \\
MCA & $8(26.7)$ & $38(39.2)$ \\
ACA/Pcom/posterior & $14(46.7)$ & \\
\hline
\end{tabular}

Values are presented as mean \pm standard deviation or number (\%). PHASES : Population, Hypertension, Age, Size of aneurysm, Earlier SAH history from another aneurysm, Site of aneurysm, SAH : subarachnoid hemorrhage, ICA : internal carotid artery, MCA : middle cerebral artery, ACA : anterior cerebral artery 
mean \pm standard deviation, while the categorical variables were rendered as numbers and percentages. Independent $\mathrm{T}$ or $\times 2$ testing were used to determine the factors related to high ruptured risk aneurysms. $p<0.05$ potential independent variables in the univariate analysis were included into multivariate regression analysis using a forward process to predict aneurysms at higher risk of rupture. The variables cutoff values with the best sensitivity and specificity for differentiating high risk aneurysms from intermediate-low aneurysms were identified by assessing the receiver operating characteristic (ROC) curve.

\section{RESULTS}

From February 2016 to October 2017, a total of 133 patients with 157 intracranial aneurysms underwent MR-VWI examination in our center. We excluded 25 patients harboring 30 aneurysms, 24 of which were proved to be dissecting aneurysms, while six were excluded due to poor MRI image quality. Finally, 108 patients with 127 unruptured saccular aneurysms were enrolled for analysis. Of these patients, 74 were women and 34 were men. Mean age of the patients were $58.5 \pm$ 10.6 (range, 17-80). Sixty-four patients (59.3\%) had hypertension, while 20 patients (18.5\%) had a previous SAH from another aneurysm (Table 1).
Thirty aneurysms had PHASES score $\geq 10$ (higher risk for aneurysm rupture), and 97 aneurysms had PHASES score $<10$ (intermediate-low risk for aneurysm rupture). Univariate

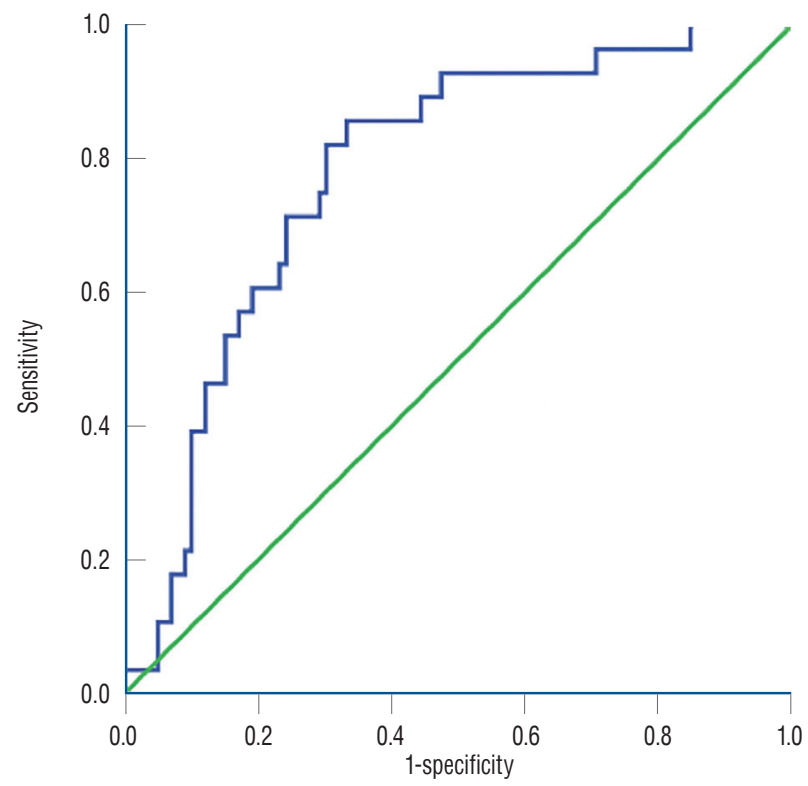

Fig. 4. High rupture risk (PHASES score $\geq 10$ ) was efficiently differentiated on ROC curve (AUC, $0.780 ; p<0.001)$. Higher WEl than cutoff value (1.04; sensitivity, 0.833; specificity, 0.67) predict high rupture risk. PHASES : Population, Hypertension, Age, Size of aneurysm, Earlier SAH history from another aneurysm, Site of aneurysm, ROC : receiver operating characteristics curve, AUC : area under the curve, WEI : wall enhancement index.

Table 2. Compare clinical rupture risk factors between high and intermediate-low risk group

\begin{tabular}{|c|c|c|c|}
\hline Parameter & High-risk $(n=30)$ & Intermediate-low $(n=97)$ & $p$-value \\
\hline Gender, female & $21(70.0)$ & $66(68.0)$ & 0.840 \\
\hline Hyperlipidemia & $6(20.0)$ & $23(23.7)$ & 0.672 \\
\hline Smoking & $6(20.0)$ & $25(25.8)$ & 0.520 \\
\hline Drinking & $4(13.3)$ & $12(12.4)$ & 1.000 \\
\hline Multiple & $9(30.0)$ & $46(47.4)$ & 0.092 \\
\hline Lobulation & $28(93.3)$ & $76(78.4)$ & 0.112 \\
\hline Daughter sac & $20(66.7)$ & $56(57.7)$ & 0.383 \\
\hline $\operatorname{Neck}(\mathrm{mm})$ & $4.5 \pm 3.3$ & $3.4 \pm 1.7$ & 0.002 \\
\hline $\mathrm{EH}$ & $30(100.0)$ & $61(62.9)$ & $<0.001$ \\
\hline Type & & & $<0.001$ \\
\hline No EH & $0(0.0)$ & $36(37.1)$ & \\
\hline Partial EH & $15(50.0)$ & $43(44.3)$ & \\
\hline Circumferential EH & $15(50.0)$ & 18 (18.6) & \\
\hline WEl & $1.6 \pm 0.6$ & $0.8 \pm 0.8$ & $<0.001$ \\
\hline
\end{tabular}

Values are presented as mean \pm standard deviation or number (\%). EH : enhancement, WEl : wall enhancement index 
analysis showed that neck $(4.5 \pm 3.3 \mathrm{~mm}$ vs. $3.4 \pm 1.7 \mathrm{~mm}$, $p=0.002)$, wall enhancement ( $100.0 \%$ vs. $62.9 \%, p<0.001)$, enhancement type $(p<0.001)$, and WEI $(1.6 \pm 0.6$ vs. $0.8 \pm 0.8$, $p<0.001)$ were significantly associated with for high rupture risk (Table 2). Compared with the partial, the circumferential type presents higher proportion of rupture risk, however, there was no statistical difference ( $25.9 \%$ vs. $45.5 \%$, $p=0.056$ ). The WEI of circumferential type presents higher than the partial ( $1.6 \pm 0.6$ vs. $1.2 \pm 0.7, p=0.011)$. However, there was no association between PHASES score and hyperlipidemia, smoking status, drinking, and aneurysms with irregular shape. Multivariate regression analysis revealed that WEI was the most important factor in predicting high rupture risk (odds ratio, 2.6; 95\% confidence interval, 1.4-4.9; $p=0.002$ ). The ROC analysis can efficiently differentiate high risk aneurysms (area under the curve, $0.780 ; p<0.001$ ) which have a reliable WEI cutoff value (1.04; sensitivity, 0.833 ; specificity, 0.67 ) predictive of high rupture risk (Fig. 4).

\section{DISCUSSION}

In this study, we prospectively compared MR-VWI detected wall enhancement extent, type and clinical risk factors between intracranial aneurysms at high and intermediate-low risk for future rupture. The neck width, wall enhancement extent including quantitative WEI and type were found to occur in aneurysms at higher risk of rupture based on PHASES score. The WEI was the independent risk factors to predict the risk of aneurysm rupture. The promising WEI cutoff value (1.04; sensitivity, 0.833 ; specificity, 0.67 ) was proposed to predict high rupture risk aneurysms.

Intracranial AWE has been proposed as an imaging marker for rupture risk assessment. Edjlali et al. ${ }^{6}$ demonstrated that circumferential aneurysmal wall enhancement was more frequently observed in unstable (ruptured, symptomatic, or undergoing morphologic modification) than stable (incidental and nonevolving) aneurysms (87\% vs. $28.5 \%$, respectively). Circumferential enhancement in ruptured aneurysms was higher than in unruptured aneurysms. Wang et al. ${ }^{29)}$ investigated 106 intracranial aneurysms with 19 ruptured aneurysms, including 10 patients with multiple aneurysms. Enhanced ratio $(>61.5 \%)$ and aneurysm with partial wall enhancement are better predictors of rupture. Nagahata et al. ${ }^{21)}$ studied 61 ruptured and 83 unruptured aneurysms, of which wall enhancement was observed in $73.8 \%$ of ruptured aneurysms and in $4.8 \%$ of unruptured aneurysms. Atherosclerosis, inflammation, neovascularization, and the presence of vasa vasorum were proposed to be possible mechanisms of wall enhancement ${ }^{13,25)}$. Miyata et al. ${ }^{19)}$ studies that vasa vasorum formation in an adventitia is associated with rupture of intracranial aneurysm. Endothelial disruption damage or inflammatory healing response could also explain this type enhancement in ruptured aneurysms ${ }^{12,18)}$. Such enhancement pattern in high rupture risk aneurysms could be associated with a larger range and greater extent of enhancement compared with intermediate-low risk aneurysms in our results. The larger wall enhancement range have an increased trend of high risk of rupture consistent with previous reported studies. However, statistical difference was not found in this present study which might be related with uneven data distribution and small sample size. The circumferential type have greater intensity than the partial. High wall enhancement extent with quantitative evaluation can predict aneurysm rupture risk. Wall enhancement might be an indicator of ruptured aneurysms for managing patients with subarachnoid hemorrhage, especially in multiple aneurysms ${ }^{8,18)}$. This enhancement would not reveal in wall itself but to the interface enhancement between wall and the surrounding brain tissue in ruptured aneurysms. Fresh thrombus in or around the ruptured point might be enhanced in these acute ruptured cases. In our study, wide-necked aneurysms were found to have a high risk of rupture. The wider neck with increased size could be explained this phenomenon. As previously demonstrated, the wall enhancement was independently associated with aneurysm size in unruptured intracranial aneurysms ${ }^{2,15,16)}$. Further prospective and pathological investigation is needed to prove the exact mechanisms of this enhancement effect.

In this present study, ROC analysis can efficiently differentiate high risk aneurysms from intermediate-low risk based on PHASES score which provides 5-year absolute aneurysm rupture risk. We concluded a reliable WEI cutoff value (1.04; sensitivity, 0.833 ; specificity, 0.67 ) which was proposed to quantitatively differentiate high risk aneurysm based on 3D MR-VWI under reference of frontal parenchymal signal intensity to predict a ruptured state. Omodaka et al. ${ }^{22)}$ reported a WEI cutoff value (0.53) to differentiate ruptured from unruptured status in circumferential enhanced aneurysms. Due 
to the signal intensity variability on high resolution-MR imaging with different parameters setting, this cutoff value cannot be generalized. However, our study definitely indicated that higher WEI are correlated to increased rupture risk. Aneurysm daughter sac assumed to be the ruptured site was locally enhanced in some aneurysms. Intracranial AWE was proposed as a characteristic of ruptured aneurysms. Previous studies used qualitative assessment to reveal that wall enhancement was more frequently occurred in ruptured aneurysm $^{6,18,21)}$. Vergouwen et al. ${ }^{27)}$ reported gadolinium enhancement of aneurysm wall on MRI predict the increased risk of aneurysm instability in the longitudinal design study. Pathophysiologically, vascular wall enhancement is related to the inflammatory reaction which plays a crucial role in the growth and rupture of intracranial aneurysms in the vascular wall $^{10,23,25)}$. In hemodynamic aspects, local changes in hemodynamic stress can activate the inflammatory reaction of endothelial cells, mononuclear cell aggregation and activation, and a variety of inflammatory molecules release from the vessel wall ${ }^{1,23,26)}$. These eventually cause the release of matrix metalloproteinases and vessel wall cells apoptosis, wall degenerative reconstruction, leading to wall thinning and aneurysm rupture ${ }^{4,17,23,26)}$. Therefore, the higher WEI predict higher PHASES score suggesting that higher WEI (>1.04) may provide additional information of aneurysm instability to improve current size-based rupture risk assessment metrics.

\section{Study limitations}

Limitations for this study are as follows. First, patient selection bias and limited number of cases from a single institution might be affecting this study consequence. Second, some confounding factors such as enhanced intra-aneurysm flow stagnation, surrounding veins, dura, venous sinus and hematoma may obscure wall enhancement. Third, the heterogeneous distribution aneurysms may be confounding factor in this study. Fourth, the causal relationship was not being concluded between the aneurysm enhancement and aneurysm rupture due to the retrospective design study. A further larger prospective study is needed to evaluate between AWE and rupture risk.

\section{CONCLUSION}

Aneurysms with higher rupture risk based on PHASES score demonstrate increased neck size, wall enhancement, and the enhancement intensity. Higher WEI in aneurysm has a predictive value for increased rupture risk.

\section{CONFLICTS OF INTEREST}

No potential conflict of interest relevant to this article was reported.

\section{INFORMED CONSENT}

Informed consent was obtained from all individual participants included in this study.

\section{AUTHOR CONTRIBUTIONS}

\author{
Conceptualization : LH, YJ, XZ \\ Data curation : LG \\ Formal analysis : GL \\ Funding acquisition : XZ \\ Methodology : YJ, HW \\ Project administration : DG, XZ \\ Visualization : $\mathrm{LH}, \mathrm{YJ}$ \\ Writing - original draft : $\mathrm{YJ}$ \\ Writing - review \& editing : FX
}

\section{ORCID}

$\begin{array}{ll}\text { Yeqing Jiang } & \text { https://orcid.org/0000-0001-7558-917X } \\ \text { Feng Xu } & \text { https://orcid.org/0000-0002-8268-0155 } \\ \text { Lei Huang } & \text { https://orcid.org/0000-0001-9199-6939 } \\ \text { Gang Lu } & \text { https://orcid.org/0000-0002-5732-0879 } \\ \text { Liang Ge } & \text { https://orcid.org/0000-0003-3706-0288 } \\ \text { Hailin Wan } & \text { https://orcid.org/0000-0001-6761-4603 } \\ \text { Daoying Geng } & \text { https://orcid.org/0000-0002-1707-1521 } \\ \text { Xiaolong Zhang } & \text { https://orcid.org/0000-0002-1206-5413 }\end{array}$

\section{- Acknowledgements}

This study was supported by the National Natural Science 
Foundation of China (81771242 and 81371308).

\section{References}

1. Aoki T, Kataoka H, Ishibashi R, Nozaki K, Egashira K, Hashimoto N : Impact of monocyte chemoattractant protein-1 deficiency on cerebral aneurysm formation. Stroke 40 : 942-951, 2009

2. Backes $D$, Hendrikse J, van der Schaaf I, Algra A, Lindgren AE, Verweij $\mathrm{BH}$, et al. : Determinants of gadolinium-enhancement of the aneurysm wall in unruptured intracranial aneurysms. Neurosurgery 83 : 719725, 2018

3. Backes D, Vergouwen MD, Tiel Groenestege AT, Bor AS, Velthuis BK, Greving JP, et al. : PHASES score for prediction of intracranial aneurysm growth. Stroke 46 : 1221-1226, 2015

4. Chalouhi N, Ali MS, Jabbour PM, Tjoumakaris SI, Gonzalez LF, Rosenwasser RH, et al. : Biology of intracranial aneurysms: role of inflammation. J Cereb Blood Flow Metab 32 : 1659-1676, 2012

5. Chan DY, Abrigo JM, Cheung TC, Siu DY, Poon WS, Ahuja AT, et al. : Screening for intracranial aneurysms? Prevalence of unruptured intracranial aneurysms in Hong Kong Chinese. J Neurosurg 124 : 1245-1249, 2016

6. Edjlali M, Gentric JC, Régent-Rodriguez C, Trystram D, Hassen WB, Lion $S$, et al. : Does aneurysmal wall enhancement on vessel wall MRI help to distinguish stable from unstable intracranial aneurysms? Stroke 45 : 3704-3706, 2014

7. Edjlali M, Guédon A, Ben Hassen W, Boulouis G, Benzakoun J, Rodriguez-Régent $C$, et al. : Circumferential thick enhancement at vessel wall MRI has high specificity for intracranial aneurysm instability. Radiology 289 : 181-187, 2018

8. Endo H, Niizuma K, Fujimura M, Sato K, Inoue T, Osawa S, et al. : Ruptured cerebral microaneurysm diagnosed by 3-dimensional fast spinecho T1 imaging with variable flip angles. J Stroke Cerebrovasc Dis 24 : e231-e235, 2015

9. Greving JP, Wermer MJ, Brown RD Jr, Morita A, Juvela S, Yonekura M, et al. : Development of the PHASES score for prediction of risk of rupture of intracranial aneurysms: a pooled analysis of six prospective cohort studies. Lancet Neurol 13 : 59-66, 2014

10. Jamous MA, Nagahiro S, Kitazato KT, Tamura T, Aziz HA, Shono M, et al. : Endothelial injury and inflammatory response induced by hemodynamic changes preceding intracranial aneurysm formation: experimental study in rats. J Neurosurg 107 : 405-411, 2007

11. Khan MO, Toro Arana V, Rubbert C, Cornelius JF, Fischer I, Bostelmann $R$, et al. : Association between aneurysm hemodynamics and wall enhancement on 3D vessel wall MRI. J Neurosurg, 2020 [Epub ahead of print]

12. Krings T, Mandell DM, Kiehl TR, Geibprasert S, Tymianski M, Alvarez $H$, et al. : Intracranial aneurysms: from vessel wall pathology to therapeutic approach. Nat Rev Neurol 7 : 547-559, 2011

13. Larsen N, von der Brelie C, Trick D, Riedel CH, Lindner T, Madjidyar J, et al. : Vessel wall enhancement in unruptured intracranial aneurysms: an indicator for higher risk of rupture? High-resolution MR imaging and correlated histologic findings. AJNR Am J Neuroradiol 39 : 1617-1621, 2018

14. Linn FH, Rinkel GJ, Algra A, van Gijn J : Incidence of subarachnoid hemorrhage: role of region, year, and rate of computed tomography: a metaanalysis. Stroke $27: 625-629,1996$

15. Liu P, Qi H, Liu A, Lv X, Jiang Y, Zhao X, et al. : Relationship between aneurysm wall enhancement and conventional risk factors in patients with unruptured intracranial aneurysms: a black-blood MRI study. Interv Neuroradiol 22 : 501-505, 2016

16. Lv N, Karmonik C, Chen S, Wang X, Fang Y, Huang Q, et al. : Relationship between aneurysm wall enhancement in vessel wall magnetic resonance imaging and rupture risk of unruptured intracranial aneurysms. Neurosurgery 84 : E385-E391, 2019

17. Marchese E, Vignati A, Albanese A, Nucci CG, Sabatino G, Tirpakova B, et al. : Comparative evaluation of genome-wide gene expression profiles in ruptured and unruptured human intracranial aneurysms. J Biol Regul Homeost Agents 24 : 185-195, 2010

18. Matouk CC, Mandell DM, Günel M, Bulsara KR, Malhotra A, Hebert R, et al. : Vessel wall magnetic resonance imaging identifies the site of rupture in patients with multiple intracranial aneurysms: proof of principle. Neurosurgery 72 : 492-496; discussion 496, 2013

19. Miyata H, Imai H, Koseki H, Shimizu K, Abekura Y, Oka M, et al. : Vasa vasorum formation is associated with rupture of intracranial aneurysms. J Neurosurg : 1-11, 2019

20. Mocco J, Brown RD Jr, Torner JC, Capuano AW, Fargen KM, Raghavan $M L$, et al. : Aneurysm morphology and prediction of rupture: an international study of unruptured intracranial aneurysms analysis. Neurosurgery $82:$ 491-496, 2018

21. Nagahata $S$, Nagahata $M$, Obara $M$, Kondo $R$, Minagawa $N$, Sato $S$, et al. : Wall enhancement of the intracranial aneurysms revealed by magnetic resonance vessel wall imaging using three-dimensional turbo spin-echo sequence with motion-sensitized driven-equilibrium: a sign of ruptured aneurysm? Clin Neuroradiol 26 : 277-283, 2016

22. Omodaka S, Endo H, Niizuma K, Fujimura M, Inoue $T$, Sato $K$, et al. : Quantitative assessment of circumferential enhancement along the wall of cerebral aneurysms using MR imaging. AJNR Am J Neuroradiol 37 : 1262-1266, 2016

23. Peña-Silva RA, Chalouhi N, Wegman-Points L, Ali M, Mitchell I, Pierce GL, et al. : Novel role for endogenous hepatocyte growth factor in the pathogenesis of intracranial aneurysms. Hypertension 65 : 587-593, 2015

24. Samaniego EA, Roa JA, Hasan D : Vessel wall imaging in intracranial aneurysms. J Neurointerv Surg 11 : 1105-1112, 2019

25. Shimonaga $K$, Matsushige $T$, Ishii D, Sakamoto S, Hosogai M, Kawasumi $T$, et al. : Clinicopathological insights from vessel wall imaging of unruptured intracranial aneurysms. Stroke $49:$ 2516-2519, 2018

26. Takemura Y, Hirata Y, Sakata N, Nabeshima K, Takeshita M, Inoue T : Histopathologic characteristics of a saccular aneurysm arising in the nonbranching segment of the distal middle cerebral artery. Pathol Res Pract 206 : 391-396, 2010

27. Vergouwen MDI, Backes D, van der Schaaf IC, Hendrikse J, Kleinloog R, 
Wall Enhancement Extent and Rupture Risk | Jiang Y, et al.

Algra A, et al. : Gadolinium enhancement of the aneurysm wall in unruptured intracranial aneurysms is associated with an increased risk of aneurysm instability: a follow-up study. AJNR Am J Neuroradiol 40 : 1112-1116, 2019

28. Vlak MH, Algra A, Brandenburg R, Rinkel GJ : Prevalence of unruptured intracranial aneurysms, with emphasis on sex, age, comorbidity, coun- try, and time period: a systematic review and meta-analysis. Lancet Neurol 10 : 626-636, 2011

29. Wang GX, Wen L, Lei S, Ran Q, Yin JB, Gong ZL, et al. : Wall enhancement ratio and partial wall enhancement on MRI associated with the rupture of intracranial aneurysms. J Neurointerv Surg $10: 566-570$, 2018 